\title{
THE INFLUENCE OF THE INITIAL DISTRIBUTION ON A RANDOM WALK
}

\author{
WOLFGANG STADJE
}

(Communicated by Daniel W. Stroock)

\begin{abstract}
Let $T_{1}, T_{2}, \ldots$ be i.i.d. random variables, $S_{n}=T_{1}+\cdots+T_{n}$; let $X$ and $Y$ be independent of $\left(T_{n}\right)_{n \geq 1}$. We study the total variation distance between the distributions of $X+S_{n}$ and $Y+S_{n}$, especially its speed of convergence to 0 in the case that some $S_{j}$ is not singular.
\end{abstract}

1. Introduction. Let $T_{1}, T_{2}, \ldots$ be a sequence of independent, identically distributed random variables and $S_{n}:=T_{1}+\cdots+T_{n}$. If $T_{1}$ is not concentrated on some lattice, $E\left(T_{1}\right)=0$ and $\sigma^{2}=E\left(T_{1}^{2}\right)<\infty$,

$$
\sigma(2 \pi n)^{1 / 2} P\left(S_{n} \in I\right) \rightarrow \lambda(I), \quad \text { as } n \rightarrow \infty,
$$

for all intervals $I \subset \mathbf{R}$, where $\lambda$ denotes the Lebesgue measure (Shepp (1964), Stone $(1965,1967)$; see also Breiman (1968, Chapter 10). In this sense $S_{n}$ is asymptotically "uniformly distributed on $\mathbf{R}$ ".

Let $X$ and $Y$ be random variables which are independent of $\left(T_{n}\right)_{n \geq 1}$. In this note we study the distance between the distributions of $X+S_{n}$ and $Y+S_{n}$, measured by means of the total variation metric

$$
\left\|P^{X+S_{n}}-P^{Y+S_{n}}\right\|=\sup _{B}\left|P\left(X+S_{n} \in B\right)-P\left(Y+S_{n} \in B\right)\right| .
$$

Here the supremum is taken over all Borel subsets $B$ of $\mathbf{R}$, and, for a random variable $U, P^{U}$ denotes its distribution. Especially in renewal theory so-called delayed renewal processes $\left(X+S_{n}\right)_{n \geq 1}$ are often considered. It is intuitively clear that the initial term $X$ can have no great influence on the asymptotic behavior of $\left(X+S_{n}\right)_{n \geq 1}$. This idea has e.g. been used in Lindvall's (1977) proof of the renewal theorem. We shall show that

$$
d_{n}^{X}:=\left\|P^{X+S_{n}}-P^{S_{n}}\right\| \leq(a E|X|+b) n^{-1 / 2},
$$

if some $P^{S_{j}}$ has an absolutely continuous component. We shall also see what happens in the case when all $P^{S_{j}}$ are singular. The constants $a, b \geq 0$ in (1.3) depend only on $P^{T_{1}}$. Clearly

$$
\left\|P^{X+S_{n}}-P^{Y+S_{n}}\right\| \leq d_{n}^{X}+d_{n}^{Y},
$$

so that (1.3) also gives an estimate for (1.2).

No assumptions about moments are needed for (1.3). We note however that if $E\left(T_{1}^{2}\right)<\infty$, we also have

$$
d_{n}^{X} \geq c n^{-1 / 2}
$$

Received by the editors March 30, 1987

1980 Mathematics Subject Classification (1985 Revision). Primary 60G50, 60F99. 
for some $c>0$, if $X \equiv x=$ const. $\neq 0$. (1.5) follows from Chebyshev's inequality which gives

$$
P\left(\left|S_{n}-n E\left(T_{1}\right)\right|<\sigma n^{1 / 2}\right) \geq(n-1) / n,
$$

where $\sigma^{2}=\operatorname{Var}\left(T_{1}\right)$. Let $x>0$; the case $x<0$ is treated similarly. (1.6) implies that there is a subinterval $I=(t, t+x]$ of $\left(n E\left(T_{1}\right)-\sigma n^{1 / 2}, n E\left(T_{1}\right)+\sigma n^{1 / 2}\right]$ satisfying

$$
P\left(S_{n} \in I\right) \geq \frac{n-1}{n}\left(\left[\frac{2 \sigma n^{1 / 2}}{x}\right]+1\right)^{-1} \geq c n^{-1 / 2} \text {. }
$$

Therefore,

$$
\left\|P^{X+S_{n}}-P^{S_{n}}\right\| \geq P\left(S_{n} \leq t+x\right)-P\left(S_{n} \leq t\right) \geq c n^{-1 / 2} .
$$

Hence if $E\left(T_{1}^{2}\right)<\infty, d_{n}^{X}$ is of the same order as $n^{-1 / 2}$.

On the other hand, if $T_{1}$ has the symmetric stable distribution with characteristic function $\exp \left(-|\zeta|^{\alpha}\right), 0<\alpha \leq 2$, the following limiting relation holds for $X \equiv x \neq 0$ :

$$
\lim _{n \rightarrow \infty} n^{1 / \alpha} d_{n}^{X}=\frac{|x|}{\pi \alpha} \Gamma\left(\frac{1}{\alpha}\right) .
$$

Thus $d_{n}^{X}$ can tend to 0 much faster than $n^{-1 / 2}$.

To see (1.8), note that the density $f_{\alpha}$ of $T_{1}$ is symmetric around 0 and unimodal and that $S_{n}$ has the density $n^{-1 / \alpha} f_{\alpha}\left(n^{-1 / \alpha} t\right)$. Hence if $x>0$,

$$
\begin{aligned}
n^{1 / \alpha}\left\|P^{x+S_{n}}-P^{S_{n}}\right\| & =\frac{1}{2} \int_{-\infty}^{\infty}\left|f_{\alpha}\left(n^{-1 / \alpha} t\right)-f_{\alpha}\left(n^{-1 / \alpha}(t-x)\right)\right| d t \\
& =\int_{-\infty}^{x / 2} f_{\alpha}\left(n^{-1 / \alpha} t\right) d t-\int_{-\infty}^{-x / 2} f_{\alpha}\left(n^{-1 / \alpha} t\right) d t \\
& =2 n^{1 / \alpha} \int_{0}^{x / 2 n^{1 / \alpha}} f_{\alpha}(s) d s \rightarrow x f_{\alpha}(0) \\
& =\frac{x}{\pi} \int_{0}^{\infty} e^{-\varsigma^{\alpha}} d \varsigma=\frac{x}{\pi \alpha} \Gamma\left(\frac{1}{\alpha}\right)
\end{aligned}
$$

where for the fourth equation Fourier inversion is used. For $x<0,(1.8)$ follows by symmetry.

II. The proof. Some further notation is necessary. $\mu * \nu$ denotes the convolution of the signed measures $\mu$ and $\nu$, and $\mu^{* i}:=\mu * \cdots * \mu$ ( $i$ factors). $\varepsilon_{x}$ is the point mass at $x \in \mathbf{R}$.

THEOREM. Assume that $P^{S_{j}}=\alpha Q_{1}+(1-\alpha) Q_{2}$ for some $j \in \mathbf{N}$ and $\alpha \in(0,1]$, where $Q_{1}$ and $Q_{2}$ are probability measures and $Q_{1} \ll \lambda$. Then there are constants $a, b \geq 0$ depending only on $P^{T_{1}}$ such that

$$
\left\|P^{X+S_{n}}-P^{S_{n}}\right\| \leq(a E|X|+b) n^{-1 / 2} .
$$

ProOF. Let $q:=d Q_{1} / d \lambda$. By decomposing $Q_{1}$ into $Q_{1} 1_{\{q \leq N\}}$ and $Q_{1} 1_{\{q>N\}}$ we see that $Q_{1}$ can be assumed to have a bounded Lebesgue density. Then $Q_{1} * Q_{1}$ has a continuous Lebesgue density which of course must exceed some $\varepsilon>0$ on some interval $I$. Therefore $Q_{1} * Q_{1}$ can be written as $\beta R+(1-\beta) Q^{\prime}$, where $R$ denotes 
the rectangular distribution on $I, \beta>0$ and $Q^{\prime}$ is some probability measure. Hence we can assume that

$$
P^{S_{j}}=\gamma R+(1-\gamma) Q
$$

for some $j \in \mathbf{N}, \gamma \in(0,1]$ and some probability measure $Q$. Since

$$
\begin{aligned}
\left\|P^{X+S_{n}}-P^{X}\right\| & \leq \sup _{B} \int\left|P\left(x+S_{n} \in B\right)-P\left(S_{n} \in B\right)\right| d P^{X}(x) \\
& \leq \int\left\|P^{x+S_{n}}-P^{S_{n}}\right\| d P^{X}(x),
\end{aligned}
$$

it suffices to prove that for some $a, b \geq 0$

$$
\left\|P^{x+S_{n}}-P^{S_{n}}\right\| \leq(a|x|+b) n^{-1 / 2} \quad \text { for all } x \in \mathbf{R} .
$$

Let $n=i j+k$, where $k \in\{0,1, \ldots, j-1\}$ and $j$ satisfies (2.2). Then by (2.2),

$$
\begin{aligned}
\left\|P^{x+S_{n}}-P^{S_{n}}\right\| & =\left\|\left[(\gamma R+(1-\gamma) Q)^{* i} * \varepsilon_{x}-(\gamma R+(1-\gamma) Q)^{* i}\right] * P^{S_{k}}\right\| \\
& \leq\left\|(\gamma R+(1-\gamma) Q)^{* i} *\left(\varepsilon_{x}-\varepsilon_{0}\right)\right\| \\
& =\left\|\sum_{l=0}^{i}\left(\begin{array}{l}
i \\
l
\end{array}\right) \gamma^{l}(1-\gamma)^{i-l} R^{* l} Q^{*(i-l)} *\left(\varepsilon_{x}-\varepsilon_{0}\right)\right\| \\
& \leq \sum_{l=0}^{i}\left(\begin{array}{l}
i \\
l
\end{array}\right) \gamma^{l}(1-\gamma)^{i-l}\left\|R^{* l} *\left(\varepsilon_{x}-\varepsilon_{0}\right)\right\| .
\end{aligned}
$$

We have twice used the inequality $\|\mu * \nu\| \leq\|\mu\|$ which is valid for arbitrary signed measures $\mu$ and probability measures $\nu$. Now we split the sum at the righthand side of (2.5) into two terms: the sums over all $l$ for which $|l-\gamma i|>\gamma i^{3 / 4}$ or $|l-\gamma i| \leq \gamma i^{3 / 4}$, respectively. The first sum is, by Chebyshev's inequality, not larger than

$$
\begin{gathered}
\sum_{\substack{0 \leq l \leq i \\
|l-\gamma i|>\gamma i^{3 / 4}}}\left(\begin{array}{l}
i \\
l
\end{array}\right) \gamma^{l}(1-\gamma)^{i-l} \leq \frac{\gamma(1-\gamma) i}{\left(\gamma i^{3 / 4}\right)^{2}} \\
=\gamma^{-1}(1-\gamma) i^{-1 / 2} \leq K n^{-1 / 2}
\end{gathered}
$$

for some constant $K$, because $i$ is the integer part of $n / j$ and thus of the same order as $n$. The other sum can be estimated from above by

$$
\sup \left\{\left\|P^{x+\widetilde{S}_{l}}-P^{\widetilde{S}_{l}}\right\| \mid \gamma\left(i-i^{3 / 4}\right) \leq l \leq i\right\}
$$

where $\widetilde{S}_{1}:=U_{1}+\cdots+U_{l}$ for i.i.d. random variables $U_{1}, U_{2}, \ldots$ distributed according to $R$. Therefore the assertion will be proved, if we can show that

$$
\left\|P^{x+\widetilde{S}_{\imath}}-P^{\widetilde{S}_{l}}\right\| \leq(a|x|+b) l^{-1 / 2} .
$$

The density $f_{l}$ of $\widetilde{S}_{l}$ is given in Feller (1971), p. 28. It is unimodal and symmetric around $l u$, if $u$ is the center of the interval $I$. It is clear that one can, without loss of generality, assume that $u=0$. 
Now it is easily seen that if $x>0$,

$$
\begin{aligned}
\left\|P^{x+\widetilde{S}_{l}}-P^{\widetilde{S}_{l}}\right\| & =\frac{1}{2} \int_{-\infty}^{\infty}\left|f_{l}(t)-f_{l}(t-x)\right| d t \\
& =\int_{-\infty}^{x / 2}\left(f_{l}(t)-f_{l}(t-x)\right) d t \\
& =\int_{-(x / 2)}^{x / 2} f_{l}(t) d t=P\left(\left|\widetilde{S}_{l}\right| \leq x / 2\right) .
\end{aligned}
$$

Let $\Phi$ and $\varphi$ be the distribution function and the density of $N(0,1)$. Then $\Phi(y) \leq \frac{1}{2}+\varphi(0) y=\frac{1}{2}+(2 \pi)^{-1 / 2} y$ for all $y>0$. By the Berry-Esseen inequality (Feller (1971), p. 542) we obtain

$$
\begin{aligned}
P\left(\left|\widetilde{S}_{l}\right| \leq \frac{x}{2}\right) & \leq 2 \Phi\left(\frac{x}{2 \sqrt{l \operatorname{Var}\left(U_{1}\right)}}\right)-1+2 \frac{3 E\left|U_{1}\right|^{3}}{l^{1 / 2}\left[\operatorname{Var}\left(U_{1}\right)\right]^{3 / 2}} \\
& \leq l^{-1 / 2}\left[\left(2 \pi \operatorname{Var}\left(U_{1}\right)\right)^{-1 / 2} x+6 E\left|U_{1}\right|^{3}\left[\operatorname{Var}\left(U_{1}\right)\right]^{-3 / 2}\right] .
\end{aligned}
$$

The case $x<0$ is treated similarly. (2.8) is proved.

Finally we consider the case when all $P^{S_{n}}$ are singular with respect to Lebesgue measure. Assume that

$$
\sum_{n=1}^{\infty} P^{S_{n}} \perp \lambda
$$

Then we assert that

$$
P^{x+S_{n}} \perp P^{S_{n}} \quad \text { for all } n \in \mathbf{N}
$$

holds for Lebesgue-almost all $x \in \mathbf{R}$. For suppose on the contrary that

$$
\left\|P^{x+S_{n}}-P^{S_{n}}\right\|<1
$$

for some $n \in \mathbf{N}$ and all $x \in A_{n}$, where $A_{n} \subset \mathbf{R}$ satisfies $\lambda\left(A_{n}\right)>0$. Then there is an $N \in \mathbf{N}$ such that $0<\lambda\left(B_{n}\right)<\infty$ for $B_{n}:=A_{n} \cap[-N, N]$. Define the probability measure $P_{n}$ on $\mathbf{R}$ by

$$
P_{n}(B):=\lambda\left(B_{n}\right)^{-1} \int_{B_{n}} P^{x+S_{n}}(B) d x
$$

Then $P_{n} \ll \lambda$ and

$$
\left\|P_{n}-P^{S_{n}}\right\| \leq \lambda\left(B_{n}\right)^{-1} \int_{B_{n}}\left\|P^{x+S_{n}}-P^{S_{n}}\right\| d x<1 .
$$

Thus $P^{S_{n}}$ is not singular with respect to $P_{n}$ and, consequently, has an absolutely continuous component.

\section{REFERENCES}

L. Breiman (1968), Probability, Addison-Wesley, Reading, Mass.

W. Feller (1971), An introduction to probability theory and its applications. II, 2nd ed., Wiley, New York.

T. Lindvall (1977), A probabilistic proof of Blackwell's renewal theorem, Ann. Prob. 5, 482-485. 
L. A. Shepp (1964), A local limit theorem, Ann. Math. Statist. 35, 419-423.

C. J. Stone (1965), A local limit theorem for multidimensional distribution functions, Ann. Math. Statist. 36, 546-551.

(1967), On local and ratio limit theorems, Proc. Fifth Berkeley Sympos. Math. Statist. and Prob., vol. II, pp. 217-224, Univ. of California Press, Berkeley, Calif.

FACHBEREICH MAThematik/INFORMATIK, UNIVERSität OSNABRÜCK, D-4500 OSNabrüCK, FRG, Albrechtstr. 28, West Germany 